\title{
Catastrophe Theory: A unified paradigm for rangeland ecosystem dynamics
}

\author{
JEFFREY A. LOCKWOOD AND DALE R. LOCKWOOD
}

\begin{abstract}
Authors are with the Department of Plant, Soil, and Insect Sciences, University of Wyoming, Laramie 82071; and Department of Mathematics, University of Arizona, Tucson 85718, respectively.
\end{abstract}

\begin{abstract}
Rangeland ecologists have elucidated 2 apparently distinct processes underlying rangeland dynamics. In some cases, disturbed or recovering rangelands move through a gradual, continuous series of changes which has been termed succession. In other instances, rangeland dynamics are typified by sudden, discontinuous changes in the vegetation, and this has been called state-and-transition. Catastrophe theory is a mathematical framework designed for the study of discontinuous phenomena, but it also generates models that permit continuous dynamics. Based on available literature, it appears that rangeland ecosystems conform to the mathematics of catastrophe theory. Rangelands exhibit the 5 essential symptoms of catastrophe systems: modality (distinct conditions or states of existence), inaccessibility (conditions which are very unstable), sudden changes (relatively rapid movement between states), hysteresis (processes associated with degradation or recovery are not readily reversible by simply inverting the sequence of events), and divergence (relatively small changes in initial conditions can result in dramatically different outcomes with time). Catastrophe theory has been successfully used to model rangeland grasshopper population dynamics, and it appears that many of the same control variables affecting insects (e.g., temperature and precipitation) may also underlie vegetative community dynamics. Application of catastrophe theory to empirical data sets will require relatively long-term but low-intensity research efforts.
\end{abstract}

Key Words: nonlinear models, succession, state-and-transition

Range management depends on accurate models of the dynamics which underlie the extractable resources of the world's rangelands. In this regard, there are 2 competing, conceptual models that have been used to explain rangeland ecosystem dynamics. The older model is that of range succession (Clements 1916, Sampson 1919, Dyksterhuis 1949). This concept calls for smooth, continuous, and reversible changes along a gradient of ecological states. The underlying theory depends on ecosystems having predictable, repeatable processes of development from a disturbed state through 1 or more seral stages and finally reaching a climax condition.

The alternative view recognizes the concept of discontinuous, irreversible changes, the nature of which may not be easily predictable over the long-term. Although this concept is not new, it has only recently been explored in terms of understanding rangeland dynamics with the development of the state-and-threshold model of Laycock (1991). In this paradigm, rangeland ecosystems do not necessarily return to an original or climax state following disturbance. Thus, eliminating the cause of a particular change in the system (e.g., reducing grazing or ending a drought) may not result

\footnotetext{
The authors wish to thank J. Dodd and W. Laycock (University of Wyoming) for critical review of this manuscript.

Manuscript accepted 17 Jan. 1993.
}

in a predictable sequence of events that return the system to its original condition.

Recent work (Dodd 1992, Friedel 1991, Laycock 1991, Westoby et al. 1988) has suggested a dichotomy between the 2 doninant conceptual models used to describe rangeland condition dynamics. The nature of these 2 paradigms (the continuous, reversible, predictable dynamics of the succession model versus the discontinuous, irreversible, unpredictable dynamics of the state-and-thresholc model) results in 2 significant difficulties with the current state of ecological modeling in range management.

First, there appears to be a dilemma with respect to resolving the applicability of the succession and state-and-threshold models to any particular system. In classical scientific terms, it appears that the state-and-threshold paradigm excludes the possibility of an underlying continuous process inherent in the succession paradigm. It has not been suggested that the older model is without applicability (Dodd 1992, Westoby et al. 1989); rather, there are some demonstrable conditions under which it does not appear to function. As such, unless there is a single model that captures both continuous and discontinuous dynamics, range scientists are in the unenviable position of having to choose between these 2 paradigms (Dodd 1992). Such a choice is confounded by what appears to be little empirical evidence to determine which system is most likely to apply in any given ecosystem. Although Schlatterer (1989) developed a model that allows both successional (reversible) and irreversible changes, it did not clearly integrate the state-and-threshold model; the model did not include a means by which one might know (except retrospectively) which process would occur in a particular system; and it did not provide a coherent explanation of why both processes were possible.

Second, conceptual models (i.e., the current formulations of the succession and state-and-threshold systems) are extremely valuable initial tools for organizing, defining, describing, and categorizing; but they have serious limitations and must eventually mature into a mathematical framework to realize their full potential. Conceptual models are virtually always post factum descriptio.s of what has happened, and they provide relatively little information with respect to forecasting and prediction. As such, they are a starting point for, but do not in themselves constitute, an adequate theoretical base for a scientific discipline. Without an underlying mathematics to define the model, qualitative descriptions often given rise to a condition of multiple paradigms, which is considerably less valuable than a unified theory of system dynamics. Finally, a lack of mathematical rigor prevents the formulation of clear research questions with which to validate or dismiss a particular model. Without a mathematical system, the "testing" of a model becomes a process of accumulating retrospective case histories.

A solution to these 2 problems of conceptual models in range ecology must include both a unification of the succession model with the state-and-threshold model and accomplish this integra- 
tion through an established mathematical system. Although range scientists are aware of the ecological literature related to discontinuous systems, there is no widespread understanding of the associated mathematical theories which may be used to solve the dilemma of multiple, conceptual paradigms. It is the purpose of this paper to introduce the field of catastrophe theory and to explain how this theory may unify the existing range ecology paradigms with a single mathematical theory.

\section{Catastrohe Theory}

Catastrophe theory was developed by Thom (1972) to provide a theoretical framework for studying discontinuous phenomena in otherwise continuous systems. In some ways the original designation of the dynamics as "catastrophic" is unfortunate because this term is colloquially used to describe extreme and negative changes. In the mathematical usage (Thom 1972), the term refers to processes which are manifested as sudden, quantum changes in the state of the system (e.g., the shift of an animal's behavior from attack to submission, the perceptual shift which occurs with many optical illusions, or the changes in the state of an embryo during development [Saunders 1980]). Zeeman (1972, 1976, 1978) subsequently popularized the theory and suggested a wide spectrum of applications in the social and biological sciences. Despite the initial interest in catastrophe theory among ecologists, it has been the physical scientists and engineers who have made the greatest use of the mathematics. The irony in this situation was elucidated by Gilmore (1981), who observed that the physical sciences do not ultimately need catastrophe theory because the underlying equations for relevant dynamics already exist and their properties will eventually be discovered. Rather, it is in the life sciences, where the underlying equations are far from known, where catastrophe theory may have its greatest and most numerous applications. Although Gilmore (1981) went on to predict that catastrophe theory would remain largely in the realm of the physical and engineering sciences, the theory has garnered some attention in range management. Jameson (1988) noted the potential applicability of the theory to modelling ecosystem dynamics, but he overlooked one of the critical properties of catastrophic systems (inaccessibility, see Catastrophic Symptoms). More importantly, he viewed the theory as simply another tool, and failed to recognize the broader implications which allow for a unification of the successional and state-and-threshold concepts. Most recently, catastrophe theory has been used to gain insight to and generate accurate predictions of the dynamics of rangeland grasshopper populations (Lockwood and Lockwood 1991). It is largely from this work that we derive the following description of the theory.

Catastrophe theory deals directly with the properties of mathematical singularities (i.e., sudden changes or discontinuities) in systems which possess a smooth, underlying potential. The necessity of a smooth function arises from the fact that we are interested in the origin of discontinuities, and nothing is gained if we merely assume they are built into the dynamics. That is, if we are free to arrange the discontinuities as we please (as with some conceptual models) our models become simply descriptive accounts, without a clear rationale. As such, catastrophe theory is particularly appropriate for the study of systems whose inner workings are unknown and for situations in which reliable observations relate to the discontinuities. Thus, the theory appears to have significant potential in unifying the 2 paradigms of range ecology.

Catastrophe theory addresses the specific conditions in which discontinuous behavior (characteristic of the state-and-threshold paradigm) can emerge from an underlying smooth potential (characteristic of the succession paradigm). Perhaps the only drawback of the theory in this regard is that it is tailored to address the singularities rather than the continuous function. However, there currently appears to be greater interest in the discontinuities in rangeland ecosystems, and this dynamic may be the more important feature of an effective management strategy (Ellis and Swift 1988).

Catastrophe theory describes the qualitatively different discontinuities in a given system that can be completely specified by 1 or more state variables (e.g., rangeland condition classes) and that is driven by 1 or more independent control variables (e.g., temperature, rainfall, and fire). The theory is constrained by a practical assumption. In Thom's (1975) definitions of elementary catastrophes, the control variables are restricted to 5. As Saunders (1980) points out, this assumption is less of a restriction than it might seem, because we can omit any independent control variables which do not have a significant effect on the discontinuity (remember that it is the sudden change, not the underlying continuous dynamic, that is the principal object of interest). Moreover, if more than 5 control variables are critically involved in the discontinuity of a system, then the analysis becomes intractable.

The simplifying elegance of catastrophe theory lies in the discovery that the number of qualitatively different discontinuities that can occur depends not on the number of state variables, which may be extremely large, but on the number of control variables, which is generally small (Saunders 1980). In range ecology there has been some rational reduction of the state variables into a limited set of functional groups (e.g., Friedel et al. 1988), and this process facilitates the application of catastrophe theory by defining the essential states of a system. However, even with this simplification of state variables, to model large and complex systems (such as rangelands composed of numerous soil types and dozens of plant species, each with its own set of herbivores and abiotic tolerances) by conventional, mechanistic means would require us to develop as many differential equations as there are state variables which specify the system, supply initial conditions, solve the equations, and then try to comprehend the solutions. Even if the rangeland ecosystem can be categorized into a relatively small number of states, mechanistic models of the dynamics are likely to be intractable. This may be particularly true if virtually every rangeland system has a unique set of regulatory mechanisms, which seems to be the case based on the models of Westoby et al. (1989). As noted by Lockwood and Lockwood (1991), a typical rangeland ecosystem is likely to be governed by more than $\mathbf{2 0 , 0 0 0}$ direct or primary relationships, and inclusion of indirect effects results in over 1 billion interactions between the living components of the system. If only $1 \%$ of the direct relationships are mechanistically important, we will need to develop and parameterize $\mathbf{2 0 0}$ mathematical expressions. With very few assumptions, catastrophe theory makes it possible to predict much of the qualitative behavior of a system without knowledge of these interactions, much less solutions to the associated differential equations (Saunders 1980). This is not to say that ordinary differential equations may not provide a powerful tool if the mechanisms of rangeland ecology are elucidated (catastrophic behavior can arise even in simple systems of ordinary differential equations [Murray 1980]), but at this time neither the precise mechanisms not the quantitative relationships necessary to describe the mechanisms appear to be available.

\section{Fold Catastrophe}

The fold is the most simple expression of catastrophe theory. As described by Saunders (1980), the fold catastrophe occurs along a response curve of the state variable in relation to a single control variable, the result of which is a parabola defining the equilibrium surface (Fig. 1). As the state variable moves along the parabola, where $u<0$, to the point of inflexion (at the origin: 0,0 ), it "jumps" into the undefined region, where $u>0$. The 2 limitations of the fold catastrophe in its most elementary form are that there is only a 


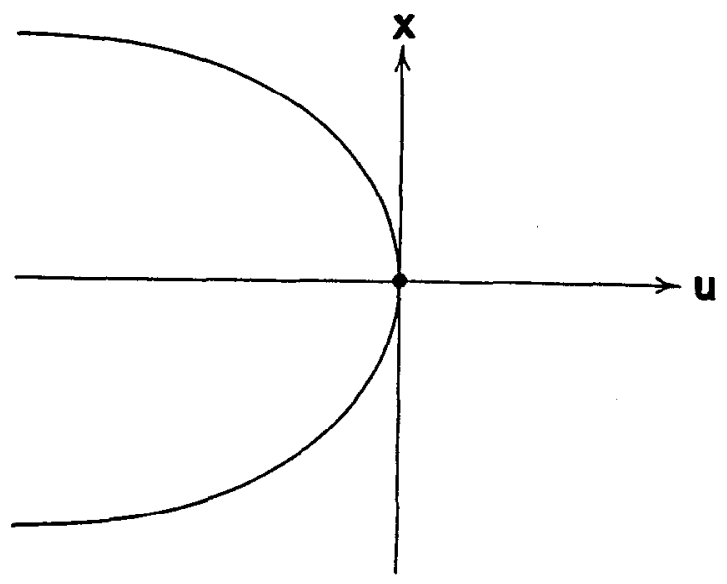

Fig. 1. The Fold Catastrophe, showing the response curve and the point of bifurcation.

single control variable, and unless we hypothesize a second fold or other modification (Saunders 1980), the dynamic is unidirectional because the state variable becomes undefined after a catastrophe. However, this model successfully demonstrates how a single expression can account for both smooth, continuous dynamics and sudden jumps.

\section{Cusp Catastrophe}

Of the 7 catastrophes that can occur with 4 or fewer control variables (Saunders 1980), the cusp catastrophe has had the greatest utility in the biological sciences (Zeeman 1976, Lockwood and Lockwood 1991). Although for some systems the overriding control may be expressed with a single variable (Lockwood and Lockwood 1988, Ellis and Swift 1988), this appears to be an exceptional condition. The utility of the cusp catastrophe may reflect the fact that it is the most parsimonious elementary catastrophe that includes more than a single control variable, and discontinuities in most living systems appear to be the consequence of more than a single factor. Thus, the sudden conversion of a dog's state from submission to attack has been modelled using the 2 behavioral parameters of rage and fear (Saunders 1980); cellular frontiers in embryology have been modelled using space and time as control variables (Zeeman 1974); prey biomass and time have been used to model predator outbreaks and crashes in chemostats (Bazin and Saunders 1979); and expressions of socio-political costs and threats have been used to understand the formation of government policy (Isnard and Zeeman 1976, Zeeman 1977). For the purpose of illustrating catastrophe theory in context of rangeland dynamics, the cusp catastrophe has the added advantage of having a solution that can be represented in 3 dimensions. While 3 and 4 variable systems can be used, if necessary (e.g., Jameson 1987), the essential features and properties are best illustrated with the cusp; rational extrapolations to the 4 and 5 dimensional solutions of more complex models should be apparent. Finally, there appears to be a number of cases in rangeland ecology where a 2-state system (e.g., arid and semiarid rangelands with distinct states represented by grassland and woodland or intact and degraded soil) having a pair of dominant control variables (Westoby et al. [1989] specifically argue that 2 control variables may very often capture the essential dynamics of rangeland systems) may be a reasonable reflection of reality (Foran et al. 1986, Friedel 1991).

The cusp catastrophe is the simplest of the bifurcation catastrophes, in which very small perturbations in initial conditions can lead to very different dynamics (see Appendix). The cusp catastrophe occurs in a response surface of a state variable in relation to 2 control variables. These latter variables are known as the normal and splitting factors. That is, across a range of values for the normal factor, any change in the splitting factor results in only smooth or "successional" changes in the state of the system (Fig. 2).

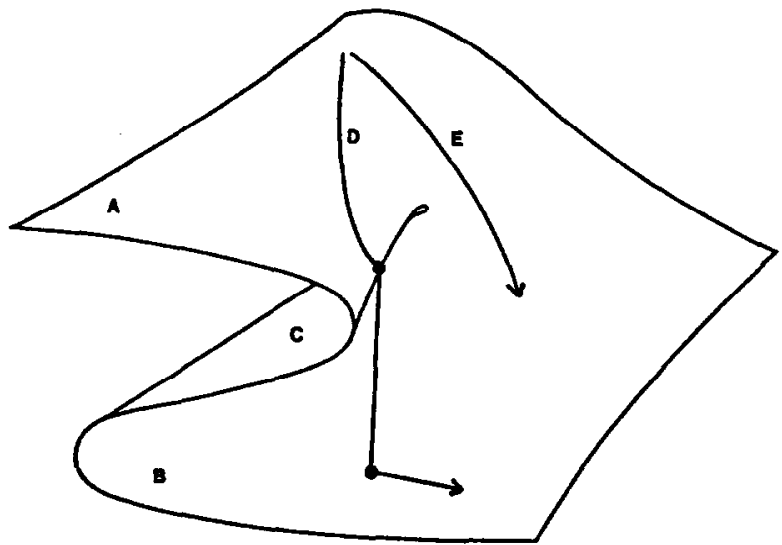

Fig. 2. The Cusp Catastrophe, showing the properties and symptoms of the model. Modality is indicated by the existence of an upper (A) and lower (B) sheet. The region of inaccessibility is shown as the infolded portion of the cusp (C). A trajectory resulting in a sudden jump from one state to another (from the upper sheet [A] to the lower sheet [B]) is indicated (D); this is the dynamic that has been previously identified in state-and-threshold models. A nearby trajectory (E) demonstrates the symptom of divergence, in which small deviations in initial conditions lead to very different behaviors. This trajectory (E) shows a process by which transition from one state to another is accomplished by a smooth, continuous dynamic, as in succession. If this trajectory (E) were to pass through the point at which the other trajectory (D) contacted the lower sheet or state (B), it would not then jump to the upper sheet $(A)$ because this point does not represent contact with the region of inaccessibility (C). This phenomenon, in which dynamics are not strictly reversible, is termed hysteresis.

However, when the normal factor falls within another range of values, the equilibrium surface which defines the state of the system is split, and changes in the other variable may give rise to discontinuous or "state-and-threshold" dynamics (Fig. 2); that is, as the control variables change and the state variable crosses the cusp edge, it will suddenly jump to the other leaf of the surface. This bifurcation is of particular interest because it represents a catastrophic change in the system (e.g., a change of state from one condition class or habitat type to another). The nature of the control variables is not predetermined. Either intrinsic variables within a feedback system (e.g., herbivore grazing or decomposition rates) or extrinsic variables indirectly modifying or outside of a feedback loop (e.g., weather or herbicide application) may be appropriate (Zeeman 1974, 1977; Isnard and Zeeman 1976; Bazin and Saunders 1979).

\section{Catastrophic Symptoms}

Because application of catastrophe theory to an ecological problem is not a simple task nor are all ecological dynamics catastrophic in nature, it is useful to determine if a particular system is likely to be accurately described with the theory. In this regard, theoretical and empirical evidence (Zeeman 1978; Lockwood and Lockwood 1988, 1991) have established that catastrophic systems exhibit a unique set of symptoms. The 5 symptoms or flags of the cusp catastrophe have been clearly observed and documented in rangeland ecosystem dynamics.

The first symptom is modality, which refers to the existence of the system in distinct states. These states are represented by the 2 surfaces of the cusp (Fig. 2) or the multiple surfaces of more complex catastrophic models. It is clear that range ecologists have identified modality. Multiple ecological states appear to be com- 
mon throughout the world (Ellis and Swift 1988, Westoby et al. 1989, Friedel 1991, Laycock 1991). While Westoby et al. (1989) recognized both states and transitions, they appear to have excessively complicated their models by defining even transitory conditions as states. The concept of discrete states of the system and the transitions between states has been clarified in recent studies. Most recently, Friedel (1991) refers to the existence of discontinuous environmental change between "alternative states" and graphically defines distinct states found in arid open woodland range. Laycock (1991) adopted and clearly applied the "state-and-threshold" concept to describe ecosystem dynamics and in so doing ascribed to the notion of modality.

The next symptom is inaccessibility, or the occurrence of unstable equilibria or thresholds (Berryman 1982), represented by the infolded region of the cusp (Fig. 2). Again, Westoby et al. (1980), and most recently both Friedel (1991) and Laycock (1991), have clearly and coherently argued for the existence of unstable equilibria. The "cup-and-ball" anology has been used to capture the concept of thresholds which separate steady states (Hurd and Wolf 1974). Indeed, the cup-and-ball conceptualization can be found both in Laycock's (1991) model of rangeland dynamics and in Saunder's (1980) explanation of elementary catastrophes. What is expressed in the "cup-and-ball" is the perceived existence of singularities and attractors or local minima of the potential.

The third condition indicating a catastrophic system is the existence of sudden jumps, which are seen when a trajectory reaches an edge of the cusp (the area of inaccessibility) and jumps to the alternative sheet or condition (Fig. 2). The occurrence of quantum leaps or discontinuities is explicitly developed by Friedel (1991), who suggests that environmental change on rangeland can be "discontinuous" (Bradbury et al. 1983/4). This symptom is also implicitly supported by Laycock (1991) in the concept of thresholds through which a system passes rapidly between 2 steady states.

The next system is hysteresis, which refers to the property in which a trajectory associated with a jump in one direction (e.g., from the upper to the lower sheet of the cusp in Fig. 2) is different from the trajectory resulting in a jump in the opposite direction. That is, a trajectory passing through the point where the system falls to the lower sheet will not, at the same point, jump up to the upper sheet. Noy-Meir and Walker (1986) described hysteresis at the effect where decline and recovery follow different paths, and Friedel (1991) built on the work of Westoby et al. (1989) in adapting this property to a model of rangeland ecosystem dynamics. Friedel (1991) suggested that shifts between states of rangeland are not practically reversible without substantial human intervention, which is another formulation of the hysteresis property (see Coffin et al. 1991). Archer's (1989) study of the conversion of mesquite savannas to woodlands was used by Laycock (1991) to support the concept of hysteresis; he suggested that while heavy grazing may drive a rangeland plant community from one state to another, the simple elimination of grazing after the transition will not result in the return of the system to its original state. Indeed, Laycock (1991) deviates from the global model, which suggests that ecological systems always returns to a single climax state, by arguing that hysteresis must be considered as a component of the system.

Finally, divergence is the symptom which arises when relatively small changes in the control variables result in markedly different behaviors of the system (Fig. 2). This concept appears to be strongly supported by the work of Gleason (1926) and Egler (1954), which found that rangeland plant communities may be regulated by relatively small differences in the conditions at the time of disturbance. The work of Westoby et al. (1989) and West (1979, 1988) suggests that in at least some systems, seemingly small changes in control variables (e.g., rainfall events or light grazing) may cause a transition between distinct states. Friedel (1991) also alludes to divergence in recognizing that different communities have different susceptibilities to change. Schlatterer's (1989) contention that the particular path of vegetative change is determined by the kind, duration, and intensity of disturbance also allows the property of divergence.

\section{Advantages of Catastrophe Theory as a Framework}

Appropriate policies and management strategies are only possible if the fundamental dynamics of the rangeland system are clearly understood (Ellis and Swift 1988). While Laycock (1991) is certainly justified in arguing that we should begin with conceptual models to organize available information, it is critical for ecologists to develop these models from systems of qualitative concepts, generalizations, and assumptions (Westoby et al. 1989) to rigorously testable systems with clearly defined relationships. Laycock (1991) observed that the conceptualized models of scientists (i.e., the system of discontinuous dynamics between multiple stable states of rangeland) must be communicated to managers; but it is equally essential that ecologists communicate their conceptual models to mathematicians in order to fully realize the potential of the models. The advantages of moving from conceptual models to mathematical formulations (when such a move is, in fact, possible) include the capacity to test and validate the model, the ability to modify a known mathematical expression to meet site specific conditions, and the capacity to manage the resources using objective, quantitative measures. In that there appears to be considerable consensus among range ecologists that some hybridization of the successional and state-and-threshold models is needed, catastrophe theory offers at least 3 advantages.

First, the mathematics of catastrophe theory allows for both continuous and discontinuous changes in a system, although the latter are the primary dynamics of interest. Because the control variables may include internal feedback factors and external stressors, the elements of successional and nonequilibrial (sensu Ellis and Swift 1988) dynamics can be addressed. As such, even a single rangeland site can be modeled with allowance for both types of dynamics, and such situations appear to be possible (Ellis and Swift 1988, Laycock 1991). Next, because the mathematical framework of catastrophe theory is established, the discontinuities in the system being modeled are truly emergent from the control variables. Thus, the transitions between states in the model are not conveniently imposed (as appears to be the general case in existing, conceptual models) but emerge from known qualitative and quantitative relationships. Lastly, because catastrophe theory does not require a strict formulation of the mechanistic processes underlying the dynamics, it is possible to apply the theory to extremely complex systems whose internal workings have yet to be quantitatively elucidated. It appears that available knowledge of rangeland ecology is much more consistent with qualitative nonmechanistic efforts than with mechanistic models and their associated need for parameterization (Friedel 1991).

A final, possible advantage of catastrophe theory arises from the conceptual nature of the cusp (or more complex models). The cusp captures both the smooth, successional transitions and the discontinuous changes in a system. It may be that even if it takes some time to collect the necessary data to validate the catastrophic models, the conceptual model (Fig. 2) may prove valuable in capturing the processes that appear to occur on rangelands.

\section{Limitations of Catastrophe Theory}

In addition to limitations of catastrophe theory that arise from the nature of the mathematics (i.e., the model specifically focuses on the discontinuous events in a dynamic, there must be an underlying smooth potential or related function, and there can be no more than 4 control variables, for practical purposes), there are 
some philosophical difficulties. Catastrophe theory has been legitimately criticized (Berryman and Stanseth 1984) for being a descriptive allegory that provides little mechanistic insight into the biological phenomena that give rise to catastrophic behavior (Sussman and Zahler 1976, 1978). Because catastrophe theory does not require precise knowledge of the manner in which the control variables impact the state variables (only that the latter have important effects on the former), it can be said that the theory fails to explain the underlying phenomenological causes (Berryman and Stanseth 1984). These objections to catastrophe theory require 2 responses in context of rangeland management.

First, from a management perspective we are not immediately concerned about the mechanistic explanation of rangeland dynamics. While this argument perhaps overstates the case, it is clear that the manager needs accurate qualitative information regarding the effects of control variables on future dynamics, not a definitive ecological theory or mechanism that explains why the forecast works (Dodd 1992). Knowledge of regulatory mechanisms is undeniably valuable in developing sound range management practices, but the manager benefits from qualitative solutions (e.g., will grazing result in a conversion of grassland to woodland?), not quantitative mechanisms. Although theoretical ecologists may be somewhat frustrated by the "black box" approach of catastrophe theory, it remains a useful tool for analysis of complex systems. This leads to the second response to the above criticisms.

As Saunders (1980) points out, the advantage of catastrophe theory lies in the modeling of systems with intractably complex inner workings. So, it hardly seems to be a weakness that catastrophe theory fails to explain mechanisms that are well beyond the scope of our current understanding. For example, while we do not know the precise mechanisms through which precipitation influences each rangeland plant species (e.g., direct water uptake, promotion of fungal diseases, mediation of herbivore populations, etc.), there appears to be no question that rainfall is a dominant catastrophic control variable on some rangelands. Because there is an imminent need for prediction of state shifts due to natural and management inputs and the determination of feedback and other mechanisms for each rangeland type is a long way from completion, catastrophe theory seems to be an appropriate technique for qualitative, nonmechanistic analysis and forecasting.

\section{Application of the Theory: A Rangeland Example}

Catastrophe theory was first applied to the rangeland ecosystem by Lockwood and Lockwood (1991) in understanding and predicting weather-driven grasshopper population dynamics. Although fluctuations in grasshopper populations and weather conditions are clearly interrelated, attempts at defining linear relationships had been largely unsuccessful (Watts et al. 1982). The accuracy of grasshopper population models had been unsatisfactory due to 2 factors. First, pest infestations exhibit extremely rapid increases and decreases in density and area, and these changes are difficult to capture with continuous or linear models (Lockwood and Lockwood 1988). Second, the precise role of weather in mediating grasshopper populations is unknown (Watts et al. 1982). Although weather acts directly on grasshopper physiology, its effects are also mediated through food plant quality and quantity, predators, parasites, and diseases (Joern and Gaines 1990). Thus, a nonmechanistic model that incorporated the possibility of sudden changes in the system was needed, and catastrophe theory provided such a tool.

It was not surprising to find that grasshoppers exhibited the 5 classical symptoms of a cusp catastrophe, given that these insects are intimately linked to rangeland plant dynamics which also appear to be catastrophic (see Catastrophic Symptoms). The element of modality or distinct states is apparent from the outbreak and nonoutbreak conditions of rangeland grasshoppers. In grass- hopper populations, inaccessibility can be seen in the existen., of unstable population densities, recognized as "threatening" conditions that are likely to precede an outbreak. Sudden jumps from low-to-high or high-to-low densities or areas of infestation are the classicial population dynamics on western rangelands (Capinera 1987, Lockwood and Lockwood 1988). The symptom of hysteresis is evldent from the realization that in grasshopper populations, simple reversal of the sequence of conditions that generate an outbreak is not likely to precipitate a crash. Finally, divergence or sensitivity to initial conditions is seen in rangeland grasshopper population dynamics. Small changes in weather may result in very different population dynamics. That is, outbreaks do not require years of serious drought, and population crashes may be induced by relatively discrete periods of rainfall (Pickford and Riegert 1964). Thus, all of the symptoms expected in a catastrophic system were found in rangeland grasshopper population dynamics.

Based on this qualitative assessment of grasshopper ecology, we used 28 years of bimonthly mean temperature and total precipitation data as the 2 control variables for a cusp catastrophe model, in which the state variable was the area infested at outbreak $(\geq 9.6$ grasshoppers $\left.\mathrm{m}^{-2}\right)$ or threatening densities $\left(>3.6\right.$ grasshoppers $\mathrm{m}^{-2}$ but $<9.6$ grasshoppers $\mathrm{m}^{-2}$ ) (Lockwood and Lockwood 1991). The model was applied to 4 ecotopographic regions of Wyoming to determine the accuracy of both matching the observed, historical dynamics and predicting future conditions. With regard to matching observed dynamics, we used 3 scales of catastrophic change-a 15,30 , and $45 \%$ increase or decrease in the area of a region that was infested from 1 year to the next. With respect to predicting dynamics, data from 1988-1990 were not included in model development so that novel cases could be used for validation.

In all regions, the cusp catastrophe model generated a match to observed outbreaks and crashes at a frequency significantly $(P<0.05)$ better than chance (Lockwood and Lockwood 1991). In general, the model was most effective in regions that had the most serious history of outbreaks. The accuracy of the cusp catastrophe increased with the intensity and scale of the infestation (e.g., largescale changes [ $\geq 45 \%$ of a region] were modeled without error). In general, outbreaks were more accurately modeled than were crashes, and weather in April-May provided the best control variables. The model was also useful in forecasting. In the Platte Valley (the region of most serious grasshopper outbreaks), the actual area of infestation fell within the forecasted range in each year of validation. In the Thunder Basin (a region of moderately severe grasshopper outbreaks), the predictions were accurate in 2 of 3 cases; in 1989 an outbreak of 27,000 ha was predicted but did not occur. Thus, a cusp catastrophe model matched historical grasshopper population dynamics and effectively predicted future changes in the area of infestation.

\section{Future Directions}

Presently, catastrophe theory is a tool with demonstrable potential but no proven applications to rangeland plant ecology. The finding that this mathematical theory accurately models rangeland grasshopper population dynamics in both post facto and predictive modes (Lockwood and Lockwood 1988, 1991) using weather variables, provides some encouraging evidence that other rangeland organisms with populations largely influenced by weather can be successfully modeled with this approach. Indeed, it seems likely that the types of data necessary to use and validate this theory on rangeland plant communities are forthcoming. Westoby et al. (1989) identified the need to determine the factors which result in particular transitions. Even without access to the mathematical theory, Laycock (1991) had the insight to express the fundamental elements of catastrophe theory; he recognized the imminent need for the identification (and measurement) of state and control vari- 
ables. Fortunately, it appears that in many rangeland ecosystems 1 to 3 control variables are principally involved in the dynamics (i.e., fire, precipitation, and grazing [Ellis and Swift 1988, Westoby et al. 1989, Friedel 1991, Laycock 1991]). Indeed, Westoby et al. (1989) collapses all control variables into 2 types: natural events and management actions. The other essential components of catastrophe theory, state variables, appear to be quite highly refined and clearly defined for at least some ecosystems (e.g., Friedel et al. 1988).

The actual utility of catastrophe theory can be evaluated when data sets have been developed which include both state and relevant control variables over a reasonable period of time. This time period is critical. It must include examples of at least the principal dynamics that occur in the system and may, therefore, require several decades of data (although continuous records may not be essential). For the model to have sufficient information to construct a reasonable facsimile of the ecological events, at least 1 case (and preferably several) of each type of discontinuous event seen in the system must be available. In this regard, it is essential for the application of catastrophe theory, or even the further development of the existing conceptual models, that ecological data be collected with attention to the states and factors or control variables which likely produce these states (directly or indirectly). Thus, in developing data sets to validate catastrophe theory, the most reasonable state variables will likely be the condition classes or habitat types relevant to a particular rangeland ecosystem; and the most valuable control variables will probably include weather (temperature and precipitation), fire, and herbivore grazing intensity.

\section{Conclusions}

According to both Friedel (1991) and Laycock (1991), there is a largely insufficient existing theoretical base for the conversion of the conceptual models of rangeland ecosystem dynamics into sound management strategies. Catastrophe theory has allowed coherent synthesis and understanding of such complex systems as anorexia nervosa in humans (Zeeman 1976, Steward and Peregoy 1983), collapse of elastic structures (Zeeman 1976), and population dynamics of rangeland grasshoppers (Lockwood and Lockwood 1991). As such, it appears that this mathematical system represents the possibility of translating conceptual models into verifiable formats which may then be used in the development of management practices.

As a scientific field is studied, over time, there comes an increasing structure which is often mathematically based. Thus, physics, studied for millennia, is the most mathematical of the sciences (indeed in this century the boundaries between many mathematical subjects and physics have virtually disappeared). Chemistry was quantified next, but even today much of the biological sciences defies mathematical explanation. A portion of this apparent intractability is due to the inability of biologists to successfully assume complexity out of the systems that we study (as has been done in physics [Woodcock and Davis 1978]), and a part of the difficulty lies in the relative recency with which we have seriously studied living systems. However, a portion of the problem is the belief by some biologists that mathematics has little to contribute to problems as complex as ours. While we would hardly agree with Lord Rutherford that, "All science is either physics or stamp collecting," his observation that many subdisciplines are largely just collections of related facts rings true today. Fortunately, range science appears to be developing and adapting systematic theories of essential processes underlying the object of study. Ecological systems frequently do not allow the formation of paradigms or models based even roughly on mathematical structure when traditional tools are applied to the task. For example, the calculus is completely entrenched in continuity; discontinuity is marginalized since most physical actions (e.g., rolling balls, falling objects, etc.) are continuous. Only in the last half of this century have mathematicians developed the tools for dealing with the enormous and unique difficulties of discontinuities which face the ecologist (and demographer, sociologist, and psychologist). It is incumbent upon us to begin the dialogue by exploring the uses and limits of paradigms such as catastrophe theory.

\section{Appendix}

The cusp catastrophe arises from a system with 1 state variable and 2 control parameters. Since a scalar differential equation has equilibria as the only interesting dynamical structure (there can not be limit cycles, homoclinic orbits, strange attractors, or other interesting dynamics in 1 dimension), the bifurcations will be of the number and type of equilibria as the control parameters vary.

The cusp is the universal unfolding of the topological singularity, $x^{4}$. This means that the cusp is the simplest expansion around the singularity that exhibits all the possible dynamics present in any small perturbation of $x^{4}$. The potential function for the cusp is given by,

$$
\mathrm{V}(\mathrm{x})=0.25 \mathrm{x}^{4}+0.50 u \mathrm{x}^{2}+\mathrm{vx}
$$

Where the dynamics system is given by,

$$
\mathbf{x}^{\prime}=-\operatorname{grad} \mathbf{V}(\mathbf{x})=\mathbf{F}(\mathbf{x})
$$

So, if we consider the possible phase planes (the phase plane being a line in the case of one state variable) that can occur from (2) as u and $v$ are allowed to vary, we see that there can be 1,2 , or 3 equilibria (Fig. 3). If there are 3 equilibria, it is always the case in the cusp that the middle one is unstable and the other 2 are stable.
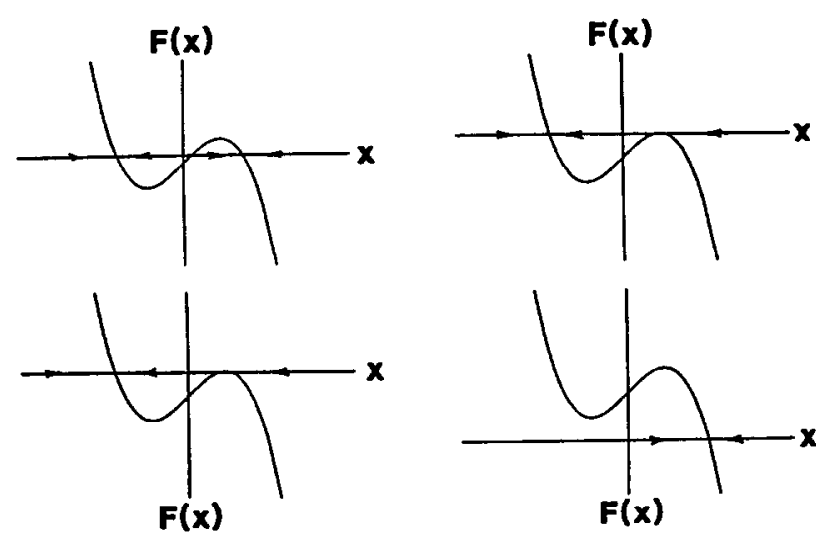

Fig. 3. Various phase portraits of the Cusp Catastrophe, showing 1, 2, and 3 equilibria.

In the case of 2 equilibria, 1 is nonhyperbolic (i.e., $F^{\prime}(x)=0$ ) and the other is hyperbolic and stable. Notice that the instability of the nonhyperbolic point is of a different form than the hyperbolic unstable point, thus giving rise to the hysteresis effect. Now, if the equilibrium manifold for (2) is generated, we get the object in Figure 2. The projection of this manifold into the $u-v$ plane yields the bifurcation diagram. The catastrophe set, which is the set of points that if crossed by the equilibrium trajectory results in a sudden jump, is given by,

$$
27 v^{2}=4 u^{3}
$$

as seen in Figure 4.

It can be seen from the mathematics of the cusp that there is an implicit assumption about the system being modelled with this catastrophe. It is necessary to assume that the state variable equilibrates very quickly, so that the data can be interpreted as being 


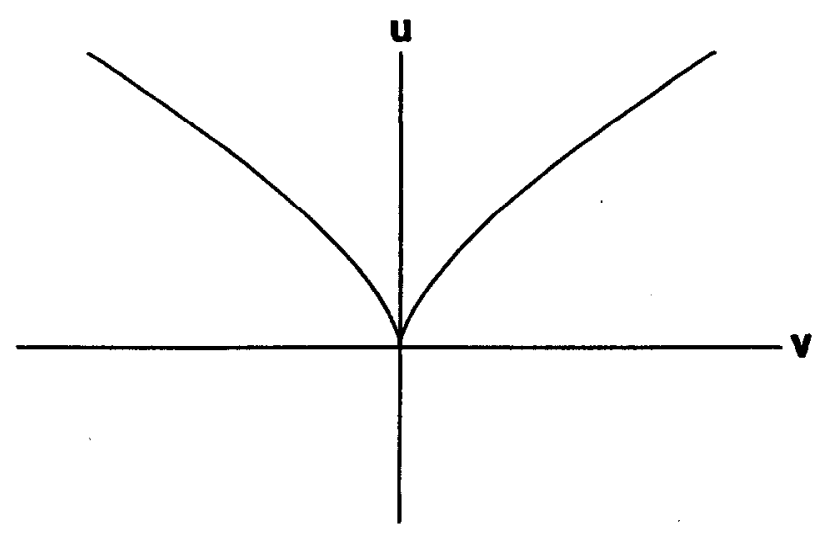

Fig. 4. Bifurcation diagram of the Cusp Catastrophe.

near the equilibrium surface. Motion near the equilibrium surface has no explicit time dependence, so without fully describing the dynamical system there can not be a deeper understanding of the asymptotic behavior of the state variable. There is also the ambiguity about the extent of the catastrophe in relation to the full dynamic. Since catastrophe theory is rooted in topology, the catastrophe can only be assumed to be a local effect of the singularity. This "localness" can only be experimentally determined, provided that the total dynamics are unknown.

\section{Literature Cited}

Archer, S. 1989. Have southern Texas savannas been converted to woodlands in recent history? Amer. Natur. 134:545-561.

Bazin, M.J., and P.T. Saunders. 1979. An application of catastrophe theory to the study of a switch in Dictyostelium discoideum. In: $\mathbf{R}$. Thomas (ed.), Kinetic logic-a Boolean approach to the analysis of complex regulatory systems. Springer, Berlin.

Berryman, A.A. 1982. Biological control, thresholds, and pests outbreaks. Environ. Entomol. 11:544-549.

Berryman, A.A., and N.C. Stenseth. 1984. Behavioral catastrophes in biological systems. Beh. Sci. 29:127-137.

Bradbury, R.H., L.S. Hammond, R.E. Reichelt, and P.C. Young. 1983/4. Prediction versus explanation in environmental impact assessment. Search 14:323-325.

Capinera, J.L. 1987. Population ecology of rangeland grasshoppers. p. 69-80. In: J.L. Capinera (ed.), Integrated Pest Management on Rangeland, a Shortgrass Prairie Perspective. Westview Press, Boulder, Colo.

Clements, F.E. 1916. Plant succession. Carnegie Inst. Wash. Pub. 242.

Coffin, D.P., W.K. Lauenroth, and I.C. Burke. 1991. Recovery of shortgrass steppe plant communities in northeastern Colorado. Abstr. Paper 82, 44th Annual Meeting, Soc. Range Mange., Washington, D.C.

Dodd, J.I.. 1992. Animal agriculture and desertification/degradation in subsaharan Africa. In: Assessment of animal agriculture in Subsaharan Africa. Winrock Int., Arlington, Virg. (In Press).

Dyksterhuis, E.J. 1949. Condition and management of rangeland based on quantitative ecology. J. Range Manage. 2:104-115.

Ellis, J.E., and D.M. Swift. 1988. Stability of African pastoral ecosystems: alternate paradigms and implications for development. J. Range Manage. 41:450-459.

Foran, B.D., G. Bastin, and K.A. Shaw. 1986. Range assessment and monitoring in arid lands: the use of classification and ordination in range survey. J. Environ. Manage. 22:67-84.

Friedel, M.H., G.N. Bastin, and G.F. Griffin. 1988. Range assessment and monitoring in arid lands: the derivation of functional groups to simplify vegetative data. J. Environ. Manage. 27:85-97.

Friedel, M.H. 1991. Range condition assessment and the concept of thresholds: a viewpoint. J. Range Manage. 44:422-426.

Gilmore, R. 1981. Catastrophe theory for scientists and engineers. John Wiley and Sons, N.Y.

Gleason, H.S. 1926. The individualistic concept of plant association. Bull. Torrey Bot. Club. 53:7-26.

Hurd, L.E., and L.L. Wolf. 1974. Stability in relation to nutrient enrichment in arthropod consumers of old-field successional ecosystems. Ecol. Monogr. 44:465 482.
Isnard, C.A., and E.C. Zeeman. 1976. Some models from catastrophe theory in the social sciences. p. 44-100. In: L. Collins (ed.), The use of models in the social sciences. Tavistock, London.

Jameson, D.A. 1987. Climax or alternative steady states in woodland ecology. p. 9-13. In: R.L. Everett (compiler), Proc. Pinyon-Juniper Conference, Reno, Nev., 13-16 Jan. 1986. USDA, Forest Serv., Gen. Tech. Rep. INT-215, Intermount. Res. Sta., Ogden, Utah.

Jameson, D.A. 1988. Modelling rangeland ecosystems for monitoring arid adaptive management. p. 189-211. In: P.T. Tueller (ed.), Vegetation science applications for rangeland analysis and management. Kluwer, Dordrecht, Netherlands.

Joern, A., and S.B. Gaines. 1990. Population dynamics and regulation in grasshoppers. p. 415-482. In: R.F. Chapman and A. Joern (eds.), Biology of grasshoppers. John Wiley and Sons, N.Y.

Laycock, W.A. 1991. Stable states and thresholds of range condition on North America rangeland: a viewpoint. J. Range Manage. 44:427-433.

Lockwood, D.R., and J.A. Lockwood. 1988. Application of catastrophe theory to population dynamics of rangeland grasshoppers. p. 268-277. In: L.L. McDonald, B.F. Manly, J.A. Lockwood, and J. Logan (eds.), Estimation and analysis of insect populations. Springer-Verlag, N.Y.

Lockwood, J.A., and D.R. Lockwood. 1991. Rangeland grasshopper (Orthoptera:Acrididae) population dynamics: insights from catastrophe theory. Environ. Entomol. 20:970-980.

Murray, J.D. 1980. Mathematical biology. Springer-Verlag, N.Y.

Noy-Meir, 1 ., and B.H. Walker. 1986. Stability and resilience in rangelands, p. 21-25. In: P.J. Joss, P.W. Lynch, and O. B. Williams (eds.), Rangeland: a resource under siege. Australian Acad. Sci. Canberra.

Pickford, R., and P.W. Riegert. 1964. The fungus disease caused by Entomophthora grylli Fr., and its effects on grasshopper populations in Saskatchewan in 1963. Can. Entomol. 96:1158-1166.

Sampson, A.W. 1919. Plant succession in relation to range management. USDA Bull. 791.

Saunders, P.T. 1980. An introduction to catastrophe theory. Cambridge Univ. Press, Cambridge, Mass.

Schlatterer, E.F. 1989. Toward a user-friendly ecosystem: myth or mirth? p. 223-227. In: D.E. Ferguson, P. Morgan, and F.D. Johnson (eds.). Proc. Land Classifications Based on Vegetation: Applications for Resource Management. USDA Forest Serv. Gen. Tech. Rep. INT-257, Intermt. Forest and Range Exp. Sta., Ogden, Utah.

Stewart, I.N., and P.L. Peregoy. 1983. Catastrophe theory modeling in psychology. Psychol. Bull. 94:336-362.

Sussman, H.J., and R.S. Zahler. 1976. Catastrophe theory as applied to the social and biological sciences: a critique. Synthese 37:117-216.

Sussman, H.J., and R.S. Zahler. 1978. Critique of applied catastrophe theory in behaviorial sciences. Beh. Sci. 23:283-389.

Thom, R. 1975. Structural stability and morphogenesis. Benjamin, Reading, Mass.

Watts, J.G., E.W. Huddleston, and J.L. Caplnera. 1982. Rangeland entomology. Annu. Rev. Entomol. 27:283-311.

West, N.E. 1979. Basic synecological relationship of sagebrush dominated lands in the Great Basin and Colorado Plateau. p. 33-4l. In: The Sagebrush Ecosystem: A Symposium. College of Natur. Resour., Utah State Univ., Logan.

West, N.E. 1988. Intermountain deserts, shrub steppes, and woodlands. p. 210-230. In: M.G. Barbour and W.D. Billings (eds.). North Amer. terrestrial vegetation. Cambridge Univ. Press, N.Y.

Westoby, M., B. Walker, and I. Noy-Meir. 1989. Opportunistic management of rangelands not at equilibrium. J. Range Manage. 42:266-274.

Woodcock, A., and M. Davis. 1978. Catastrophe theory. Dutton, N.Y.

Zeeman, E.C. 1972. A catastrophe machine. p. 276-282. In: C.H. Waddington (ed.), Towards a theoretical biology 4, essays. Edinburgh Univ. Press.

Zeeman, E.C. 1974. Primary and secondary waves in developmental biology. p. 69-161. In: S.A. Levin (ed.), Towards a theoretical biology 4, essays. Edinburg Univ. Press.

Zeeman, E.C. 1976. Catastrophe theory. Sci. Amer. 234:65-83.

Zeeman, E.C. 1977. Catastrophe theory. Addison-Wesley, Reading, Mass.

Zeeman, E.C. 1978. A dialogue between a mathematician and a biologist. Biosci. Commun. 4:225-240. 